\title{
Análise do Algoritmo Round-Robin para Balanceamento de Cargas em Redes Definidas por Software
}

\author{
Thyago Junior Rocha Dias ${ }^{1}$, Robson Gomes de Melo ${ }^{1}$, Nivaldi Calonego Junior ${ }^{1}$ \\ ${ }^{1}$ Núcleo de Redes Inteligentes e Soluções Criativas - Universidade do Estado de Mato \\ Grosso (UNEMAT) \\ Av. São João, S/N - 78.200-000 - Cáceres - MT - Brazil \\ \{thyago.dias, robinho, nivaldi\}@unemat.br
}

\begin{abstract}
The Round-Robin algorithm is widely used for process scheduling and can be applied for request scheduling in SDN networks. This paper discusses the results obtained by simulating load balancing in SDN networks using the Round-Robin algorithm. Simulation results show the effectiveness of load balancing in the SDN environment. In the scenario of network traffic congestion, the load-balanced SDN environment performed 109\% faster compared to the non-load-balanced non-SDN environment.
\end{abstract}

Resumo. O algoritmo Round-Robin é amplamente usado para o escalonamento de processo, podendo ser aplicado para o escalonamento de requisições em redes SDN. Este artigo discute os resultado obtidos por meio de simulação do balanceamento de carga em redes SDN, usando o algoritmo Round-Robin. Os resultados obtidos mostram a eficácia do balanceamento de carga no ambiente SDN. No cenário em que existiu congestionamento de tráfego na rede o ambiente SDN com balanceamento de carga atuou 109\% mais rápido em comparação ao ambiente não SDN sem balanceamento de carga.

\section{Introdução}

As redes de comunicação consistem em hosts interconectados pela infraestrutura de rede. Essa infraestrutura estabelece compartilhamento de dados entre hosts e utiliza elementos de comutação, como roteadores, switches e links de comunicação para transportar dados. De acordo com (NUNES et al., 2014), roteadores e switches em geral encontram-se como sistemas "fechados", geralmente com interfaces de controle limitadas e específicas do fornecedor, que uma vez implantado e em produção, torna-se bastante difícil para a infraestrutura de rede atual evoluir, sendo este um obstáculo quase intransponível nas redes atuais.

Segundo os dados da (CISCO, 2017), o tráfego IP global em 2016 foi de 96 EB (exabyte) por mês e quase triplicará até 2021, chegando a 278 EB mensal. O tráfego IP atingirá 232,7 EB e o tráfego IP comercial será de 45,5 EB mensalmente até 2021. No geral, o tráfego IP crescerá a uma taxa anual de 24\% de 2016 a 2021. Esse aumento causará uma sobrecarga na rede afetando o seu desempenho, o que resultará em atraso e perda de pacotes. O paradigma de Redes Definidas por Software (SDN) se consolidou neste contexto para permitir gerenciamento programável da rede e a criação de novas tecnologias e protocolos.

A principal ideia de SDN apontada por (PM; M, 2015), implica na separação desse hardware de encaminhamento do controle lógico, flexibilizando a operação de 
novos protocolos, aplicações, gerenciamento e visualização da rede. A Figura 1 ilustra a comparação da arquitetura de rede tradicional e SDN.

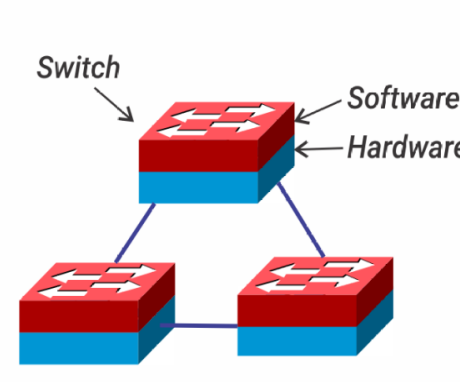

Figura 1(a) - Rede Tradicional

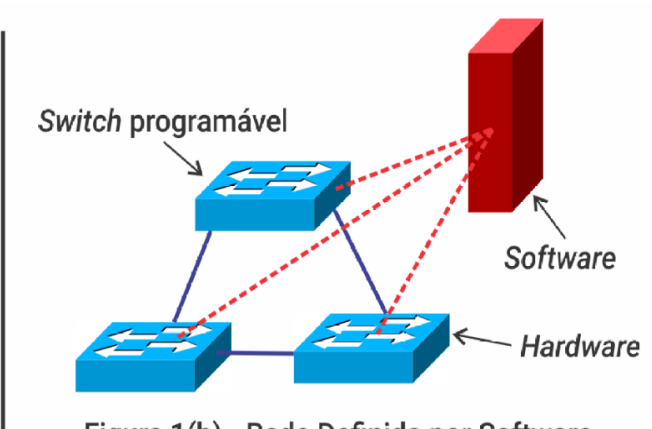

Figura 1(b) - Rede Definida por Software

Figura 1 - Rede Tradicional vs Rede Definida por Software.

A Figura 1a ilustra a Rede Tradicional, composta de hardware pouco flexível, e a Figura 1b ilustra hardware flexível em que os elementos estão integrados e formando uma SDN. A separação do controle lógico da rede permite a simulação do seu comportamento em função do algoritmo de escalonamento de recursos. Neste caso, aplica-se o algoritmo Round-Robin para avaliar o escalonamento de requisições de páginas WEB em um ambiente de rede congestionado.

\section{Trabalhos Correlatos}

Em (SILVA et al., 2017), comparam-se cinco políticas de balanceamento de carga em SDN e switch OpenFlow. O trabalho aponta que o balanceamento de carga equivale a uma forma de reduzir o tempo de respostas dos servidores. As cinco políticas comparadas são: Round-Robin, random, txbytes, cpuq-load e load/load/prev. Os resultados dos testes em data center demonstram que as políticas txbytes e cpuq-load superaram as demais. Por outro lado, load-prev foi apontada como a mais promissora ao se fazer ajustes em função do tráfego.

Em seu trabalho, (ASSUMPÇÃO et al., 2014) cria um mecanismo baseado em P2P para os switches que realizam o balanceamento de carga. Além disso, o balanceamento realiza-se por múltiplos caminhos junto à proposta de dividir os fluxos em parcelas menores. O mecanismo criado denomina-se P2PFlow e sua implementação segue o padrão OpenFlow. Os resultados obtidos pelo trabalho através de simulações em cenário multicast apresentam redução variada de $200 \%$ a $416 \%$ da utilização de banda por link.

KAUR e JYOTI (2017) apresentam uma estratégia para balanceamento com base no tráfego em SDN. Os autores discutem um algoritmo de balanceamento de carga baseado no tráfego, utilizando a abordagem de roteamento direto. O autor compara o seu algoritmo de balanceamento de carga com algoritmo Round Robin. Os resultados obtidos superaram o algoritmo Round Robin em relação ao desempenho.

Apesar dos trabalhos apresentarem estratégias para balanceamento de carga, eles não levam em consideração ambientes congestionados. Desta forma apresentam resultados que não evidenciam os benefícios que o balanceamento de carga podem prover em ambientes de rede congestionados. Assim, destaca-se a contribuição deste trabalho em avaliar o método de balanceamento de carga com algoritmo de RoundRobin em ambientes SDN congestionados, comparando seus resultados aos de um ambiente de rede não SDN sem balanceamento de carga. 


\section{Modelo Proposto}

O módulo balanceador de carga é implementado como uma aplicação em SDN, aproveitando os recursos disponíveis para minimizar o tempo de resposta e maximizar o número de transações executadas por segundo. O balanceador de carga está logicamente inserido entre o cliente e o catálogo de servidores, gerenciando o fluxo de tráfego para o rol de servidores. A carga a ser balanceada tem origem no tráfego de dados de diferentes protocolos, como Hypertext Transfer Protocol (HTTP), File Transfer Protocol (FTP), entre outros. A Figura 2 ilustra a topologia de a configuração básica de balanceamento de carga em SDN usada na construção dos cenários de simulação.

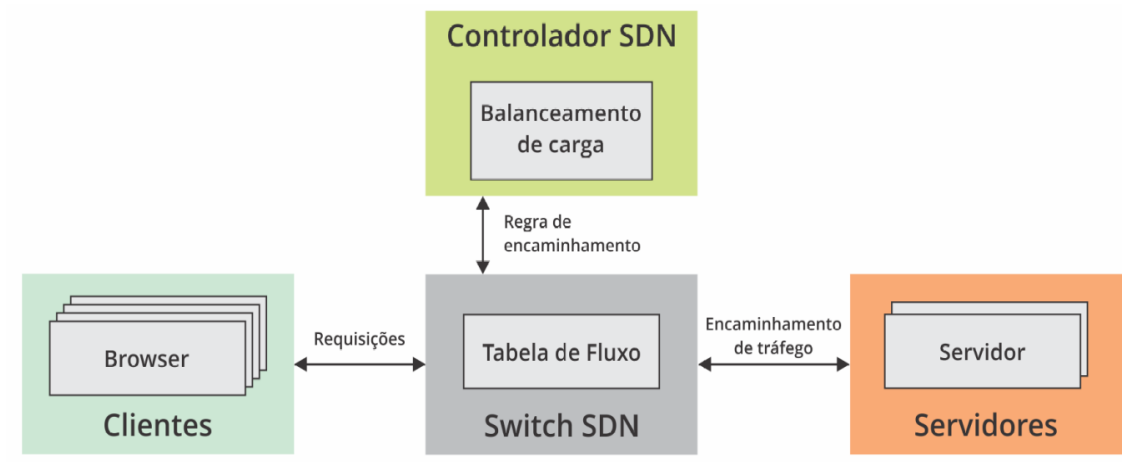

Figura 2 - Modelo SDN para Balanceamento de Carga (DIAS, 2019).

A arquitetura ilustrada na Figura 2 mostra um switch SDN conectado a um controlador e a vários servidores vinculados por meio das portas do switch. O switch SDN recebe requisições dos clientes e as redirecionam para os servidores de destino, seguindo uma regra de encaminhamento provida do controlador. Através da tabela de fluxo, o controlador insere novas entradas no switch. Para a realização do balanceamento de carga implementa-se um algoritmo como um módulo no controlador.

\subsection{Ambientes e Cenários de Simulação}

Há dois ambientes de experimento: 1 - sem rede SDN, com encaminhamento aleatório, usando quatro hosts clientes, dois servidores Web e um switch sem OpenFlow; 2 - rede SDN, com balanceamento de carga baseado em Round-Robin, um controlador POX, quatro hosts clientes, dois servidores Web e um swicth OpenFlow. A simulação dos ambientes 1 e 2 acontecem em quatro cenários distintos, conforme apresentados na Tabela 1.

Tabela 1. Cenários de Simulações

\begin{tabular}{cll}
\hline Cenário & Ambiente & Característica \\
\hline A & Sem SDN + encaminhamento aleatório & Sem congestionamento \\
B & Sem SDN + encaminhamento aleatório & Com congestionamento \\
C & SDN + balanceamento de carga & Sem congestionamento \\
D & SDN + balanceamento de carga & Com congestionamento \\
\hline
\end{tabular}

\subsection{Parâmetros de Simulação}

Para simular os cenários B e D, onde há congestionamento, foram emitidas 100 requisições HTTP dos clientes 1, 2 e 3 para o serviço à uma taxa de 10 requisições por segundo, enquanto nos cenários A e $\mathrm{C}$, sem congestionamento, apenas o cliente 4 efetuou uma requisição para o serviço HTTP. A conexão entre os dispositivos foi 
simulada como enlace de fibra ótica, com uma largura de banda de 20GB/s. O enlace de fibra foi utilizado nas simulações para verificar se em redes com alta taxa de transmissão a estratégia proposta se mostra vantajosa. A Tabela 2 sintetiza os parâmetros das simulações realizadas para os ambientes 1 e 2 para os cenários descritos.

Tabela 2. Parâmetros das Simulações.

\begin{tabular}{ll}
\hline Parâmetro & Descrição \\
\hline Computador & Notebook modelo Acer E1-572 \\
Processador & Intel i5 \\
Memória RAM & $8 \mathrm{~GB}$ \\
Sistema Operacional & Linux Ubuntu 18.04 \\
Simulador & Mininet \\
Tipo de Enlace & Fibra ótica \\
Largura de Banda & $20 \mathrm{~GB} / \mathrm{s}$ (gigabytes por segundo) \\
Tamanho da página web & $1.2 \mathrm{MB}$ \\
Controlador SDN & POX \\
Switch não OpenFlow & 1 no ambiente sem SDN com encaminhamento aleatório \\
Switch OpenFlow & 1 no ambiente SDN com balanceamento de carga \\
Número de Clientes & 4 \\
Número de Servidores & 2 \\
Browser & Chromium \\
\hline
\end{tabular}

Foram usadas as ferramentas: Mininet versão 2.2.1, para realização das simulações; HTTPerf, para gerar o tráfego na rede; o módulo SimpleHTTPServer do Python para a criação de servidores HTTP com manipuladores de solicitações GET e HEAD; o navegador Chromium, para simulações dos clientes.

\section{Resultados Obtidos}

Os resultados apresentados na Figura 3(a) mostram que o cliente sofre maior atraso para o carregamento da página no ambiente de rede sem SDN, com encaminhamento aleatório e congestionado. No cenário com congestionamento, o tempo de carregamento da página foi em média $88 \mathrm{~ms}$, aproximadamente, $220 \%$ maior do que no cenário sem congestionamento, que levou 281,5ms. Portanto, o ambiente não SDN apresentou impacto negativo no tempo de carregamento da página ao receber grande carga de tráfego em seus servidores.

Os gráficos da Figura 3(b) apresentam menor variação no tempo de carregamento entre os cenários com e sem congestionamento no Ambiente SDN, com Balanceamento de Carga. No cenário com congestionamento, o tempo de carrega mento da página foi de $134,5 \mathrm{~ms}$, aproximadamente $35 \%$ maior em relação ao cenário sem congestionamento que levou 99,5ms. Isso mostra que a variação da carga de tráfego não provocou diferença significativa no tempo de carregamento da página no ambiente com balanceamento de carga.

Os gráficos ilustrados na Figura 4 mostram o tempo de carregamento para cada uma das 30 simulações efetuadas para cada um dos ambientes, totalizando 120 simulações. 


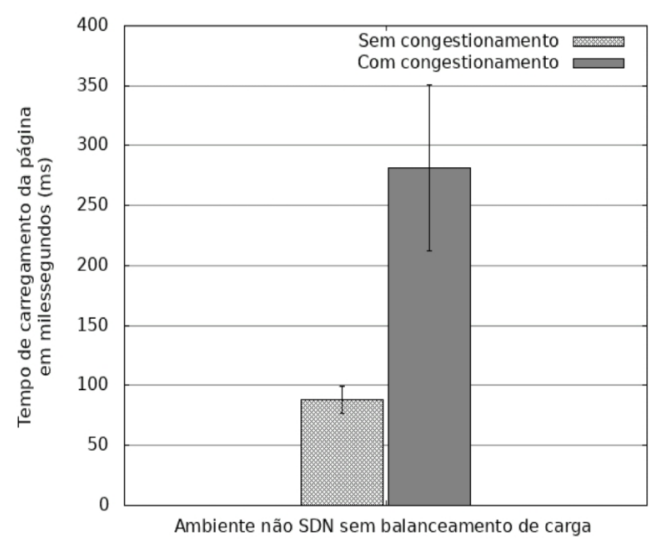

(a)

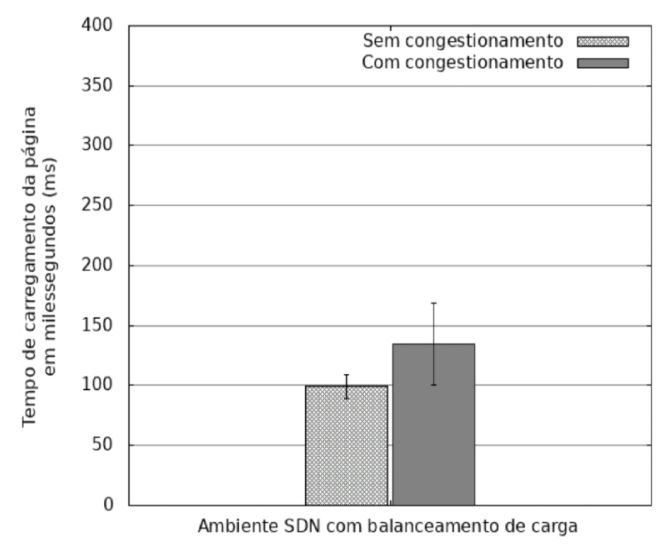

(b)

Figura 3. Simulação do tempo de carregamento.

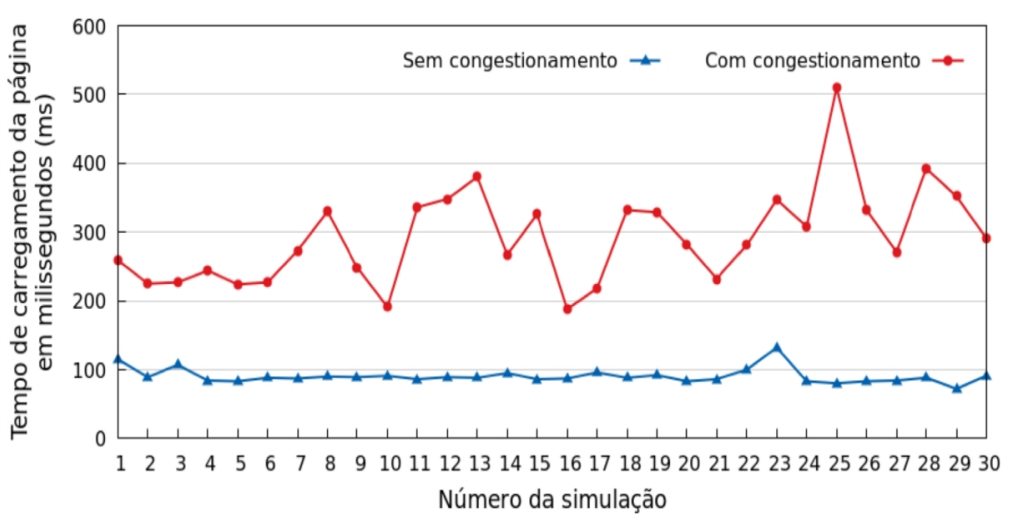

(a)

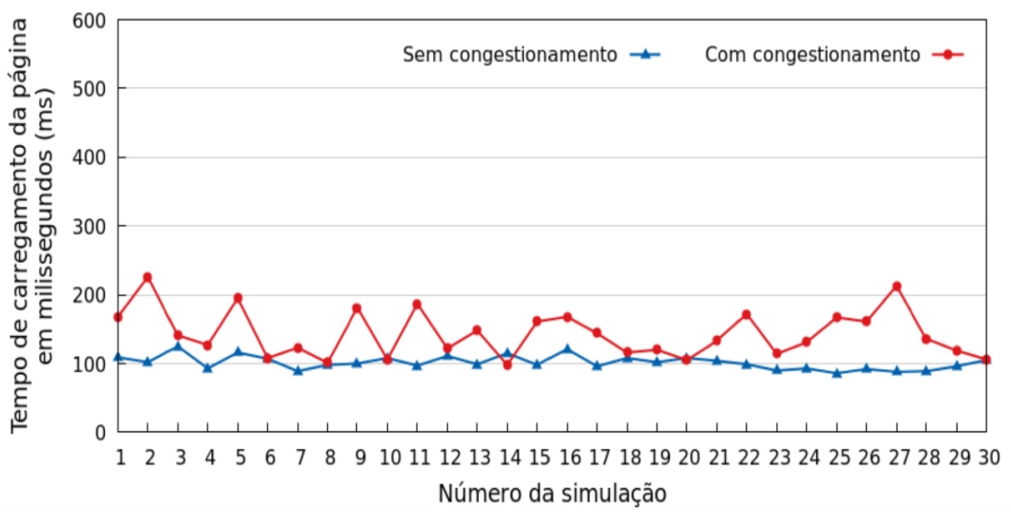

(b)

Figura 4. Tempos de carregamento das páginas.

A Figura 4(a) ilustra os resultados de simulação para o ambiente não SDN e com encaminhamento aleatório. O cenário sem congestionamento não apresenta variação significativa entre os resultados das simulações. Porém, no cenário com congestionamento, o tempo de carregamento da página apresentou variação significativa entre as simulações, apresentando valores maiores que os obtidos no cenário sem congestionamento, dado que os servidores gastam mais tempo para responder às requisições. A diferença entre os resultados apresentou-se grande, devido ao cliente ter feito a requisição da página para um servidor que estava mais congestionado e, em 
outros momento, para o servidor menos congestionado, apresentando maior oscilação entre os resultados.

A Figura 4(b) mostra o resultado das simulações para o ambiente SND, com balanceamento de carga. Nesse cenário, sem congestionamento, não há variação significativa entre os resultados. Porém, no cenário com congestionamento o tempo de carregamento da página apresentou uma leve alternância entre os resultados. Comparando como cenário sem congestionamento, o ambiente SDN com balanceamento de carga apresentou um bom resultado com congestionamento, pois não apresentou um impacto considerado negativo no tempo de carregamento da página. Sendo assim, em algumas das simulações, o tempo de carregamento congestionado foi menor do que no mesmo ambiente sem congestionamento. Isso ocorre devido à estratégia de balanceamento de carga equilibrar a carga nos servidores, evitando que um deles venha a ficar congestionado.

\section{Considerações Finais}

Os resultados obtidos nos cenários simulados com congestionamento de tráfego mostraram que a estratégia balanceamento de carga em SDN melhora, significativamente, o desempenho da rede. Comparado ao ambiente não SDN com encaminhamento aleatório, SDN com balanceamento de carga apresentou melhora de $109 \%$ no desempenho da rede com congestionamento de tráfego. Isso sustenta a afirmação de que a estratégia apresentada é fundamental para garantir o melhor desempenho da rede em ambientes com congestionamento.

\section{References}

ASSUMPÇÃO, J. H. de B. et al. Balanceamento da Carga de um Fluxo em Múltiplos Caminhos Usando Conceito de Redes Par-a-Par. IX Workshop de Redes P2P,Dinâmicas, Sociais e Orientadas a Conteúdo, 2014.

CISCO. Cisco Visual Networking Index: Global Mobile Data Traffic Forecast Update, 2016-2021 White $2017 . \quad$ Paper. $<$ https://www.cisco.com/c/en/us/solutions/collateral/service-provider/visualnetworking-index-vni/mobile-white-paper-c11-520862.html>.

KAUR, H.; JYOTI, N. Traffic Based Load Balancing in Software Defined Networking. International Journal on Computer Science and Engineering (IJCSE), 2017.

NUNES, B. A. A. et al. A Survey of Software-Defined Networking: Past, Present, and Future of Programmable Networks. IEEE Communications Surveys Tutorials, Salvador, Brasil, 2014.

PM, R.; M, D. A Study of Software Defined Networking with OpenFlow. International Journal of Computer Applications (0975 - 8887), 2015.

SILVA, E. de Britto e et al. Comparação de Políticas de Divisão de Tráfego em Data Center Empregando SDN. XXXV Simpósio Brasileiro de Redes de Computadores e Sistemas Distribuídos (SBRC), 2017.

Dias, Thyago Junior Rocha. Uma Estratégia para Avaliação da QoE a Partir do Balanceamento de Carga em SDN. Trabalho de Conclusão de Curso - Bacharelado em Ciências da Computação - Universidade do Estado de Mato Grosso, 2019. 